BMJ Open

Diabetes

Research

\& Care

\title{
Peritoneal macrophages have an impaired immune response in obesity which can be reversed by subsequent weight loss
}

\author{
Lisa Willemsen (D) , ${ }^{1}$ Annette E Neele, ${ }^{1}$ Saskia van der Velden, ${ }^{1}$ Koen H M Prange, ${ }^{1}$ \\ Myrthe den Toom, ${ }^{1}$ Cindy P A A van Roomen, ${ }^{1}$ Myrthe E Reiche (D) , ${ }^{1}$ \\ Guillermo R Griffith (D) ,' Marion J J Gijbels, ${ }^{1,2}$ Esther Lutgens, ${ }^{1,3}$ \\ Menno P J de Winther (D) ${ }^{1,3}$
}

To cite: Willemsen L, Neele AE, van der Velden S, et al. Peritoneal macrophages have an impaired immune response in obesity which can be reversed by subsequent weight Ioss. BMJ Open Diab Res Care 2019;7:e000751. doi:10.1136/ bmjdrc-2019-000751

- Additional material is published online only. To view please visit the journal online (http://dx.doi.org/10.1136/ bmjdrc-2019-000751).

Received 11 July 2019 Revised 24 September 2019 Accepted 14 October 2019
Check for updates

(c) Author(s) (or their employer(s)) 2019. Re-use permitted under CC BY. Published by BMJ.

For numbered affiliations see end of article.

Correspondence to Professor Menno P J de Winther;

m.dewinther@amsterdamumc. $\mathrm{nl}$

\section{ABSTRACT}

Introduction Obesity is recognized as a risk factor for various microbial infections. The immune system, which is affected by obesity, plays an important role in the pathophysiology of these infections and other obesityrelated comorbidities. Weight loss is considered the most obvious treatment for obesity. However, multiple studies suggest that the comorbidities of obesity may persist after weight loss. Deregulation of immune cells including adipose tissue macrophages of obese individuals has been extensively studied, but how obesity and subsequent weight loss affect immune cell function outside adipose tissue is not well defined.

Research design and methods Here we investigated the phenotype of non-adipose tissue macrophages by transcriptional characterization of thioglycollate-elicited peritoneal macrophages (PM) from mice with dietinduced obesity and type 2 diabetes (T2D). Subsequently, we defined the characteristics of PMs after weight loss and mimicked a bacterial infection by exposing PMs to lipopolysaccharide.

Results and conclusions In contrast to the proinflammatory phenotype of adipose tissue macrophages in obesity and T2D, we found a deactivated state of PMs in obesity and T2D. Weight loss could reverse this deactivated macrophage phenotype. Anti-inflammatory characteristics of these non-adipose macrophages may explain why patients with obesity and T2D have an impaired immune response against pathogens. Our data also suggest that losing weight restores macrophage function and thus contributes to the reduction of immune-related comorbidities in patients.

\section{INTRODUCTION}

Obesity is characterized by a broad spectrum of obesity-related comorbidities. The most frequently occurring comorbidities are type 2 diabetes (T2D), cancer, cardiovascular diseases, and respiratory and immune dysfunction. ${ }^{1}$ Moreover, obesity has been linked to worse clinical outcomes of bacterial infections like pneumococcal diseases, ${ }^{23}$ viral infections

\section{Significance of this study}

What is already known about this subject?

Dobesity is recognized as a risk factor for microbial infections.

- Weight loss is considered the most obvious treatment for obesity.

What are the new findings?

- We found a deactivated state of non-adipose tissue macrophages in a mouse model for obesity and type 2 diabetes (T2D).

- Weight loss could reverse this deactivated macrophage phenotype.

How might these results change the focus of research or clinical practice?

- The deactivated characteristics of these macrophages may explain why patients with obesity and T2D have an impaired immune response against pathogens.

- Our data suggest weight loss restores macrophage function and thus may lead to a reduction of immune-related comorbidities in patients.

such as dengue fever, ${ }^{4}$ and parasitic diseases like malaria. ${ }^{5}$ Furthermore, the duration of influenza A virus shedding in obese adults is increased compared with non-obese adults. ${ }^{6}$ These data indicate disturbed immune activation in obesity and a suppressed ability to fight pathogens.

Previous studies have shown that a modest weight reduction of $5 \%-10 \%$ in patients with obesity-associated complications already leads to health benefits. ${ }^{7}$ However, others indicate that obesity-induced changes, as an elevation in lipid mediators and a depletion of bone marrow-derived mesenchymal progenitor cell subpopulations, persist after weight 
loss. ${ }^{89}$ Therefore, additional treatment may be warranted to reverse all comorbidities.

Immune cell dysfunction in obese adipose tissue is well characterized. In brief, expanded adipose tissue in obesity recruits immune cells that contribute to a chronic state of low-grade inflammation and dysregulated metabolism. ${ }^{10}{ }^{11}$ Macrophages are key mediators of inflammation and insulin resistance in obesity. ${ }^{12} 13$ In adipose tissue of obese individuals, macrophage populations switch from anti-inflammatory, homeostasis maintaining cells towards a more proinflammatory phenotype with insulin desensitizing actions. However, little is known about macrophage function in non-adipose tissue in obesity, consequences of weight loss and the relation to host defense.

To define the dysregulation of non-adipose tissue macrophages in obesity and after weight loss, we analyzed thioglycollate-elicited peritoneal macrophages (PM) from mice with diet-induced obesity (DIO) and T2D. We performed systemic metabolic and immunological characterization of these mice and linked this to activation and transcriptional profiles of PMs, either under unstimulated conditions or on activation by a pathogenassociated ligand, lipopolysaccharide (LPS).

\section{RESEARCH DESIGN AND METHODS}

Mice

Six-week-old male C57BL/6J mice were purchased from Janvier Labs. Mice were housed at the Animal Research Institute Amsterdam UMC (ARIA). Mice were randomly assigned to the experimental groups in disposable IVC Rodent Caging Systems (Innovive) in groups of six mice per cage. Mice were fed a low-fat diet (LFD; $10 \%$ kcal fat, D12450B (I), Research Diets) or a high-fat diet (HFD; $60 \%$ kcal fat; D12492 (I), Research Diets) ad libitum for 10 or 20 weeks.

\section{Glucose and insulin tolerance tests}

Glucose (GTT) and insulin tolerance tests (ITT) were performed after 8-9 weeks and 18-19 weeks of the diet. Mice fasted for 5 hours for the GTT, and glucose $(1 \mathrm{mg} / \mathrm{g}$ bodyweight, Sigma-Aldrich) was injected intraperitoneally. For the ITT, mice fasted for 4 hours and insulin $(0.75$ $\mathrm{mU} / \mathrm{g}$ bodyweight, Sigma-Aldrich) was injected intraperitoneally. Blood glucose levels were measured using a Bayer Contour glucometer.

\section{Triglycerides and cholesterol measurements}

After 9 and 19 weeks of the diet, mice fasted for 4 hours before blood was collected via the tail vein. Total plasma cholesterol and triglyceride levels were quantified by enzymatic CHOD-PAP and GPO-PAP methods (Roche).

\section{Histology}

Visceral fat and liver were collected, fixed in $4 \%$ paraformaldehyde (Sigma-Aldrich) and embedded in paraffin. Four-micron sections were made and stained with H\&E. The amount of 'infiltrating' immune cells was determined by blinded examination of the sections together with an experienced pathologist and used as a measure of inflammation. Sections were assigned to a 'Low', 'Intermediate' or 'High'-grade inflammation group.

\section{Peritoneal macrophages}

After 10 or 20 weeks of the diet, six mice per group received intraperitoneal injections with 3\% thioglycollate (Fisher Scientific) to induce a sterile inflammation that attracts monocytes. Mice were euthanized by $\mathrm{CO}_{2}$ asphyxiation after 4 days. The peritoneum was flushed with ice-cold phosphate buffered saline (PBS) and cells were collected. Peritoneal cells were cultured in RPMI1640 containing $25 \mathrm{mM}$ HEPES, $2 \mathrm{mM} \mathrm{L}$-glutamine, $10 \%$ fetal calf serum, $100 \mathrm{U} / \mathrm{mL}$ penicillin and $100 \mu \mathrm{g} / \mathrm{mL}$ streptomycin (GIBCO). After 3 hours, floating cells were washed away and adherent cells $\left(\sim 95 \% \mathrm{CD} 1 \mathrm{~b}^{+} \mathrm{F} 4 / 80^{+}\right.$ macrophages, online supplementary figure S1) were stimulated with or without $100 \mathrm{ng} / \mathrm{mL}$ LPS (Sigma) for 3 or 24 hours or $20 \mathrm{ng} / \mathrm{mL}$ interleukin-4 (IL-4; Peprotech) for 24 hours. Thioglycollate-injected mice were only used for PMs and not for other analysis.

\section{Cytokine and nitric oxide measurement}

After sacrifice, whole blood was collected via the retroorbital vein in 0.5 M EDTA (Invitrogen) containing tubes. Plasma and supernatant cytokines were detected using an electrochemiluminescence assay. A V-PLEX Proinflammatory Panel 1 mouse kit (MSD) was used to measure tumor necrosis factor (TNF), interferon gamma (IFN $\gamma$ ), C-X-C motif chemokine ligand 1 (CXCL1) and IL-10. Data were acquired using a MESO QuickPlex SQ 120 plate reader (MSD). Nitric oxide (NO) production was assessed by a Griess reaction (Sigma-Aldrich) according to the supplier's protocol.

\section{Flow cytometry}

To measure surface marker expression, $1.5 \times 10^{5} \mathrm{PMs}$ were seeded per well in a 96-well plate and stimulated with $100 \mathrm{ng} / \mathrm{mL}$ LPS or $20 \mathrm{ng} / \mathrm{mL}$ IL-4 for 24 hours. After stimulation, PMs were detached with citrate buffer $(17 \mathrm{mM}$ tri-Sodium citrate dihydrate and $135 \mathrm{mM}$ potassium chloride in $\mathrm{H}_{2} \mathrm{O}$ ). Fc receptors of PMs were blocked with anti-CD16/CD32 (eBioscience) for $15 \mathrm{~min}$ at room temperature (RT). Fluorescent-labeled antibodies targeting CD11b (eBioscience), F4/80 (eBioscience), CD64 (BioLegend), CD71 (BD Pharmingen) and CD86 (eBioscience) were incubated for $20 \mathrm{~min}$ at RT. Stained cells were resuspended in PBS with $0.5 \%$ bovine serum albumin and $2.5 \mathrm{mM}$ EDTA and measured on a Beckman Coulter CytoFLEX and analyzed with FlowJo software V.10. Debris and doublets were excluded using forward and side scatter. CD11b+ andF4/80+ cells were considered as PMs.

\section{RNA sequencing}

Total RNA was isolated from PMs using the RNeasy Mini Kit (QIAGEN) with DNase treatment. RNA (700 ng) was used for Illumina library construction. RNA amplification, cDNA generation, and adaptor ligation were performed 
using the KAPA mRNA HyperPrep Kit (Roche) following the manufacturer's instructions. Samples were pooled, diluted to $10 \mathrm{nM}$ and sequenced single end on an Illumina HiSeq 4000 instrument (Illumina) to a depth of \pm 20 million reads with a length of 50 base pairs.

\section{Bioinformatics}

Reads were aligned to the mouse genome mm10 by STAR 2.5.2b with default settings. ${ }^{14}$ Binary alignment map (BAM) files were indexed and filtered on MAPQ $>15$ with SAMTools 1.3.1. ${ }^{15}$ Raw tag counts and reads per kilobase million (RPKM) per gene were summed using HOMER2's analyzeRepeats.pl script with default settings and the -noadj or -rpkm options for raw counts and RPKM reporting. ${ }^{16}$ Differential expression was assessed using the DESeq2 Bioconductor package in an R V.3.4.3 programming environment with gene expression called differential with a false discovery rate (FDR) $<0.05$ and a median RPKM $>1$ in at least one group. ${ }^{17}$ Presented RPKM values were tested using one-way analysis of variance (ANOVA) followed by Bonferroni's post hoc comparisons test. Pathway enrichment analysis was performed using Metascape. ${ }^{18}$ Gene Ontology biological processes were selected for the analysis. Upstream regulator analysis was performed using Ingenuity Pathway Analysis software (QIAGEN).

\section{Weighted gene coexpression network analysis}

A weighted correlation network was created using the weighted gene coexpression network analysis (WGCNA) $\mathrm{R}$ software package. ${ }^{19} 20$ Transcripts with a median RPKM $>1$ in at least one group of unstimulated PMs were selected for WGCNA. For network construction, an adjacency matrix was calculated with a soft-thresholding power of 8 . Signed adjacency was used for clustering and the signed hybrid model was selected as the network type. Next, to calculate the connection strength between all transcript pairs, a topological overlap measure (TOM) dissimilarity matrix (1-TOM) was used for average hierarchical clustering. Module eigengenes were calculated by summarizing the first principal component of the modules. Further, the correlation between the transcript expression profile and the module eigengene, also known as the module membership, was calculated. Next, the module-trait association was used to identify clinically relevant modules related to bodyweight (trait). For this, the correlation between the module eigengene and bodyweight was used. The gene significance (GS) measure between the expression profile and trait was calculated for every transcript and allows identification of proteins, which are strongly associated with the trait bodyweight. Hub genes, which are strongly associated with the trait bodyweight $(\mathrm{GS}>0.7)$ and with a module membership $>0.7$, were selected for the creation of a functional protein association network. The search tool for the retrieval of interacting genes/proteins (STRING) database was used for the creation of this network with high confidence $(0.7){ }^{21}$

\section{Statistical analysis}

For all experiments, five to seven mice were used per group. Outliers were identified with the robust regression and outlier detection (ROUT) method with $\mathrm{Q}=1 \%$ and subsequently removed. Normality of the data was tested using D'Agostino-Pearson and Shapiro-Wilk tests. Data were further analyzed by one-way ANOVA, two-way ANOVA, Kruskal-Wallis tests, unpaired t-test or MannWhitney test using GraphPad Prism V.7.03 (GraphPad Software). Tukey and Sidak multiple correction tests were applied when necessary. $\mathrm{P}<0.05$ was considered significant. Data are presented as means \pm SD.

\section{RESULTS}

\section{Weight loss reverses classical DI0 and T2D characteristics}

To study HFD-induced consequences followed by weight loss, we used dietary interventions to investigate mouse PMs. Male C57BL/6 mice were fed a HFD or LFD for 10 weeks. After 10 weeks, a subset of the mice was sacrificed. Next, a part of the residual HFD mice was switched to a LFD for another 10 weeks (HFD-LFD). The remaining mice continued with their original diet (figure 1A). After 2 weeks, the HFD mice gained significantly more weight compared with LFD mice (figure 1B). From 1 week after the diet shift, there was no significant bodyweight difference remaining between HFD-LFD and LFD mice. GTT and ITT showed significantly increased blood glucose levels at several time points in fasted HFD mice after 8-9 weeks (online supplementary figure S2A,B) and 18-19 weeks of the diet (figure 1C,D). There was no difference in blood glucose levels between LFD and HFD-LFD mice at any time point. Fasting plasma triglyceride and cholesterol levels were significantly increased in HFD mice after 8 weeks (online supplementary figure S2C) and 18 weeks (figure $1 \mathrm{E}$ ) and recovered completely after the diet shift. To summarize, HFD induces various obesity and T2D characteristics which disappear after weight loss.

In obesity, expanded adipose tissue contributes to a state of systemic low-grade inflammation. ${ }^{10} 11$ To confirm this chronic inflammatory state and to determine whether this state was reversible by weight loss, various plasma cytokines and chemokines were measured and immune cell quantity was analyzed in liver and visceral fat. TNF, CXCL1, and IL-10 were significantly increased after 20 weeks of HFD (figure 1F). All cytokine and chemokine levels recovered after weight loss. Adipose tissue of HFD mice contained enlarged adipocytes with an increased number of immune cells (figure 1G,H). After weight loss, adipocyte size decreased and additional immune cells disappeared. Similar effects were seen for hepatic immune cell infiltration and lipid accumulation (figure 1I,J). To conclude, weight loss repairs the HFD-induced chronic inflammatory state. 

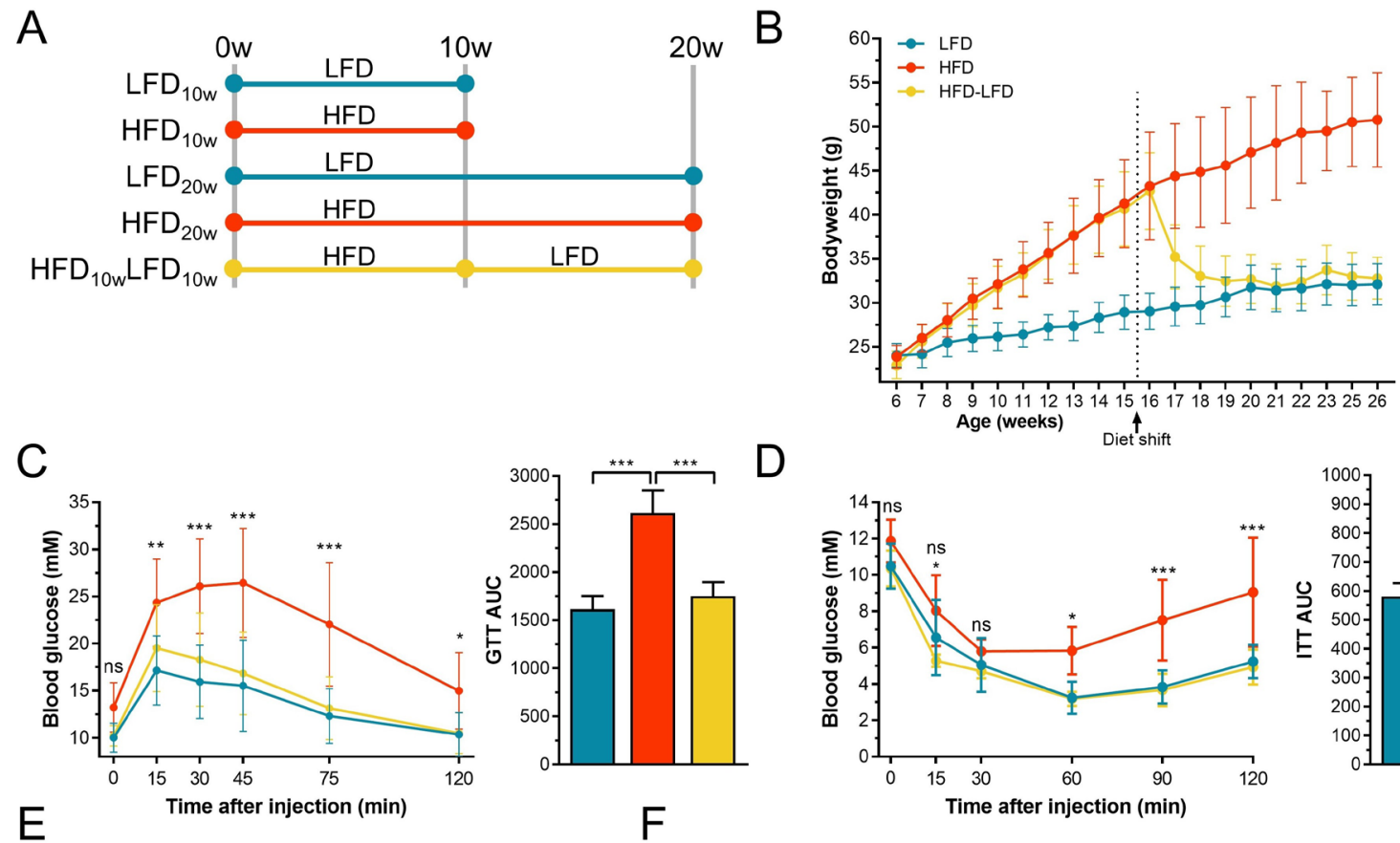

G
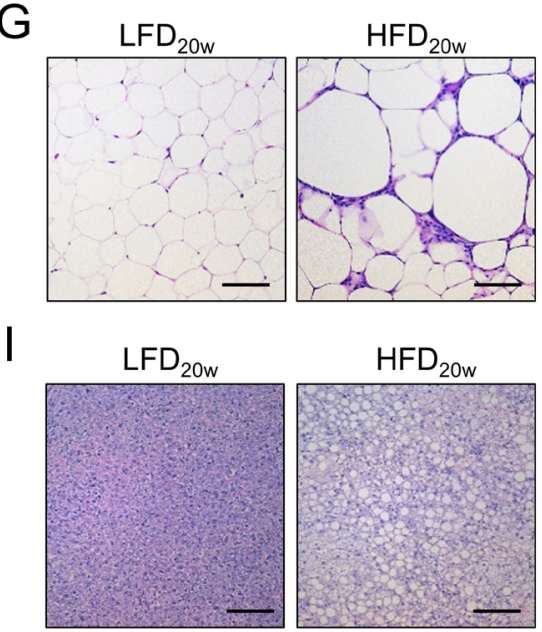
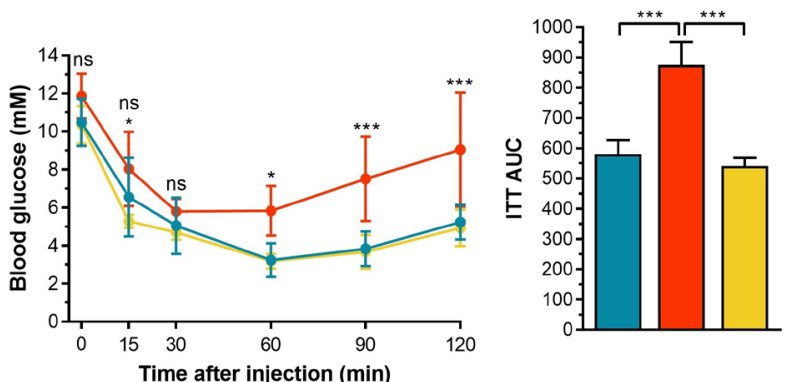

TNF
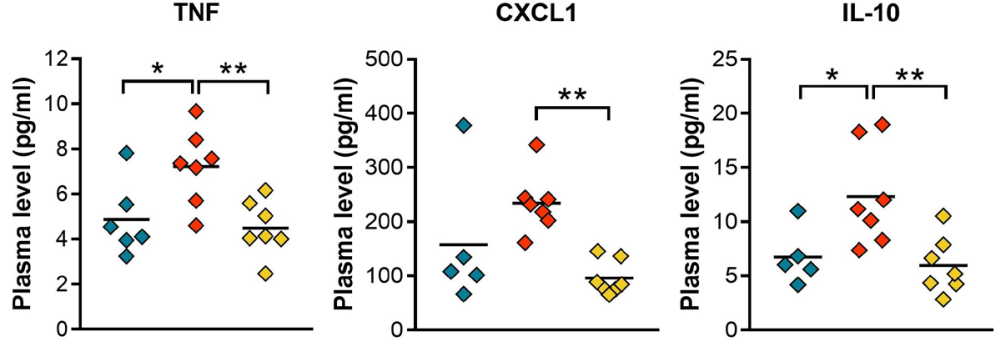

$\mathrm{H}$

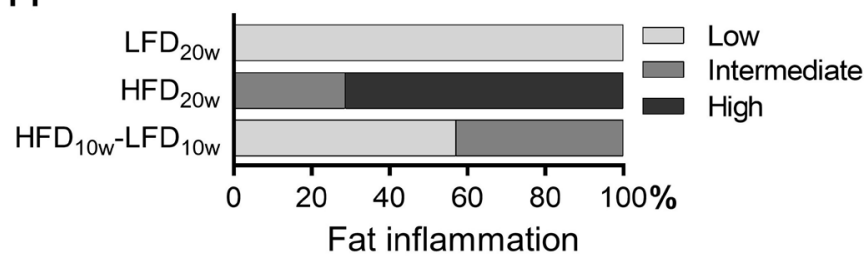

$J$

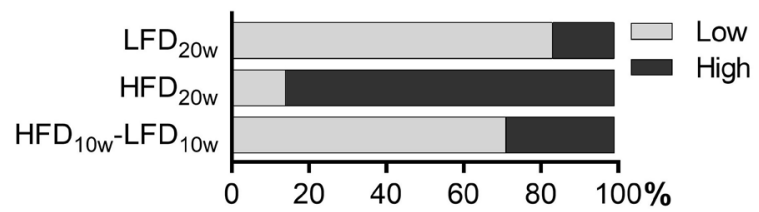

Liver inflammation

Figure 1 Diet-induced obesity (DIO) and type 2 diabetes (T2D) characteristics were reversed by subsequent weight loss. (A) Male C57BL/6 mice were fed a LFD $(n=24)$ or HFD $(n=42)$ diet for 10 weeks when 12 LFD and 14 HFD mice were sacrificed.

Fourteen of the remaining HFD mice were shifted to a LFD for 10 weeks. The rest of the mice continued with their original diet.

(B) Mouse bodyweight was measured once a week. (C) Blood glucose levels were measured over time after 5 hours of fasting and subsequent intraperitoneal injections of glucose. Glucose area under the curve (AUC) was calculated per group. (D) Blood glucose levels were measured over time after 4 hours of fasting and subsequent intraperitoneal injections of insulin. Glucose AUC was calculated per group. (E) Fasting plasma triglycerides and cholesterol levels. (F) Plasma cytokines (TNF, CXCL1, and IL-10) were measured using an electrochemiluminescence assay. (G) H\&E stained visceral fat sections. Scale bar length: $100 \mu \mathrm{m}$. (H) H\&E stained fat sections were scored for the count of infiltrating immune cells as a measure of inflammation. (I) H\&E stained liver sections. Scale bar length: $100 \mu \mathrm{m}$. (J) H\&E stained liver sections were scored for the count of infiltrating immune cells as a measure of inflammation. Error bars represent SD. AUC, area under the curve; CXCL1, C-X-C motif chemokine ligand 1; GTT, glucose tolerance test; HFD, high-fat diet; IL-10, interleukin-10; ITT, insulin tolerance test; LFD, lowfat diet; TNF, tumor necrosis factor. 
HFD primes PMs to a deactivated state that disappears after weight loss

To determine whether the macrophage response against pathogens was affected by obesity, we examined the transcriptional profiles of PMs, either under unstimulated conditions or on LPS-induced activation. As a baseline, the LPS response of PMs from LFD mice was analyzed using RNA-seq. After administration of LPS, 4654 genes were significantly upregulated and 4636 genes were significantly downregulated $(\mathrm{FDR}<0.05$; online supplementary figure S3A). As expected, transcriptional levels of important immune regulators such as $I l 1 b$, NO synthase 2 (Nos2), Il6, and Ifng were significantly induced after LPS exposure. Next, the transcriptomes of LPSstimulated PMs of LFD mice were compared with those of HFD mice to assess the impact of obesity on bacterial infection. We found 347 significantly upregulated genes and 407 significantly downregulated genes in HFD PMs compared with LFD PMs (FDR $<0.05$; figure 2A). Subsequently, we analyzed which part of the HFD-affected
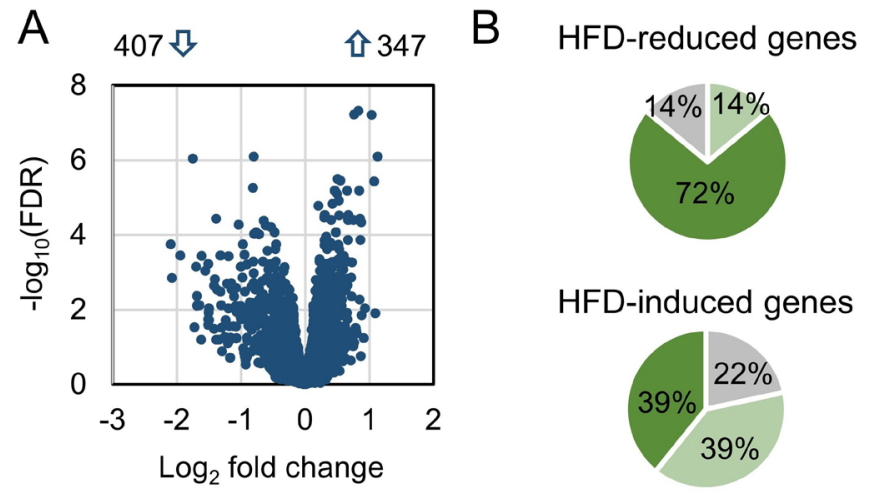
- Downregulated by LPS
- Upregulated by LPS
Not affected by LPS

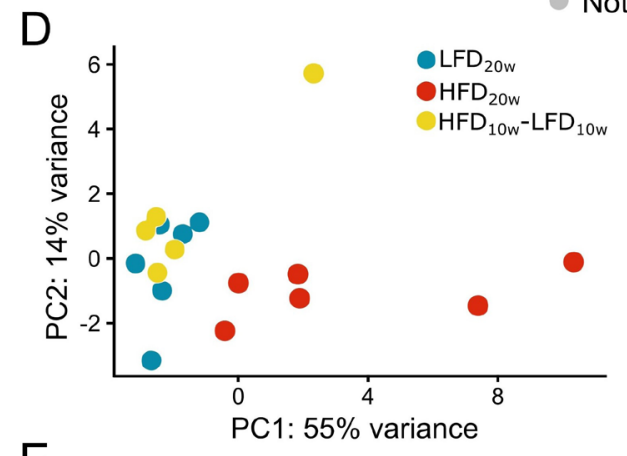

$\mathrm{E}$

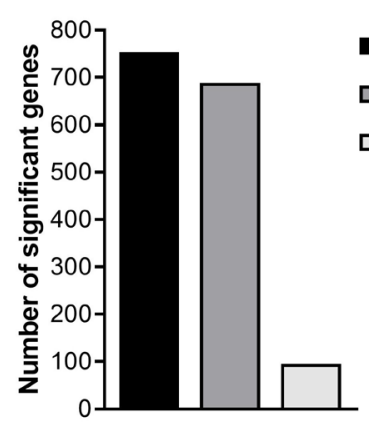

C

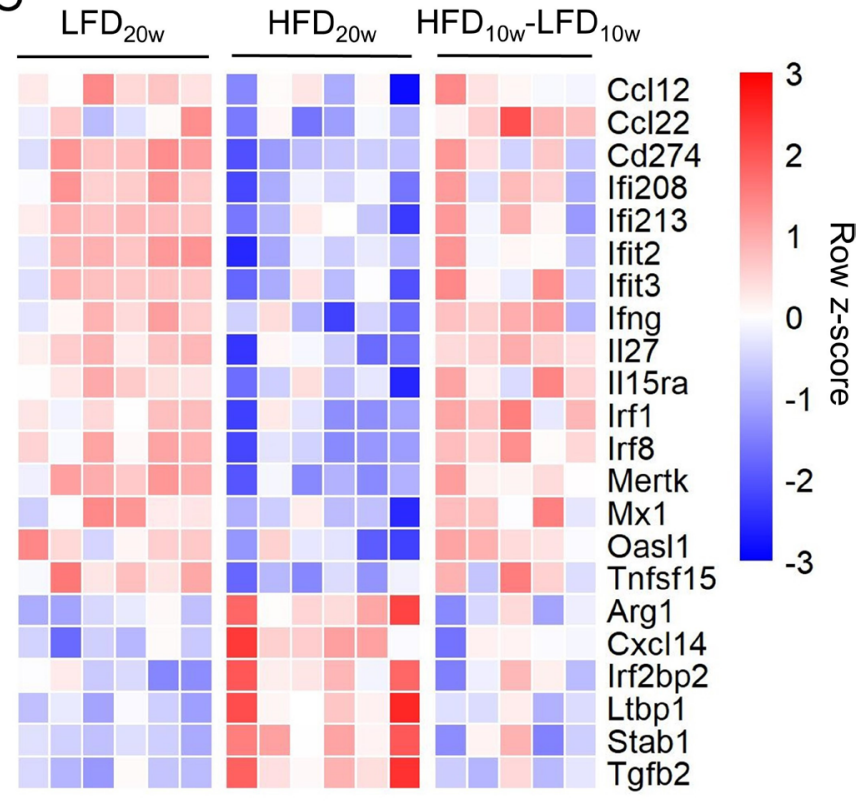

Figure 2 Transcriptomic profiles of peritoneal macrophages (PM) indicated an impaired immune response caused by HFD which disappeared after weight loss. (A) Volcano plot of the gene expression of LPS-stimulated PMs of HFD versus LFD mice. (B) Pie charts of HFD-reduced and HFD-induced genes (FDR<0.05) and the overlap with the normal (LFD) LPS response. (C) Heatmap with the row Z-score of significantly differentially (FDR $<0.05)$ regulated immune response genes of RNA-seq data of LPS-stimulated PMs. (D) Principal component analysis (PCA) of each biological RNA-seq replicate of LFD, HFD, and HFDLFD. (E) The number of significantly differentially regulated genes per comparison (FDR $<0.05)$. (F) Top five pathways of Gene Ontology pathway enrichment analysis of the top 95 differentially expressed genes of each comparison from (E). FDR, false discovery rate; HFD, high-fat diet; LFD, low-fat diet; LPS, lipopolysaccharide. 
genes are at play in the control response to LPS under LFD conditions and whether these differentially regulated transcripts are enhanced or dampened in the LPS response. For instance, genes that are significantly upregulated by LPS administration in LFD mice may be significantly less induced in HFD mice which indicates a dampened LPS response. Interestingly, we found that $72 \%$ of the genes reduced by HFD dampened the LPS response, while $14 \%$ were not affected by LPS administration (figure 2B). Besides, 39\% of the genes induced by HFD also dampened the LPS response, while 22\% were not changed by LPS exposure. Our data thus suggest a predominant suppression of the LPS response of PMs in DIO and T2D. Genes included in this suppression encompassed highly relevant proinflammatory regulators like interferon regulatory factor 8 (Irf8), Ifng and chemokine (C-C motif) ligand 12 (Ccl12) (figure 2C). Moreover, HFD induced upregulation of classical anti-inflammatory mediators such as arginase 1 (Argl) and transforming growth factor beta $2(T g f b 2)$. The LPS-induced gene expression of the genes depicted in figure $2 \mathrm{C}$ is shown in the online supplementary figure S3B. Interestingly, the effects on these and other highly relevant inflammation controlling genes completely reversed after the diet shift (figure 2C). Focusing on the reversibility of HFDaffected gene expression, we found that the principal component analysis reveals two separated clusters: one cluster that consists of overlapping LFD and HFD-LFD samples, which is moderately separated from a cluster which contains samples from the HFD PMs (figure 2D), indicating reversibility on diet switch. Moreover, there were only 95 significantly differentially regulated genes between the LFD versus HFD-LFD PMs after LPS exposure, while 689 and 754 genes were differentially regulated between HFD versus HFD-LFD and HFD versus LFD PMs (figure 2E). Pathway enrichment analysis on the top 95 differentially regulated genes of each comparison clearly showed that the immune response of PMs is affected by HFD (figure 2F). This enrichment immunerelated pathway did not exist in LFD versus HFD-LFD PMs and thus again showing a reversible profile. Under unstimulated conditions, samples clustered similarly (online supplementary figure S3C). Seven hundred and fifty-five unique genes were significantly differentially expressed between HFD and LFD without LPS exposure $($ FDR $<0.05)$. Six hundred and eighty-three genes were found to be significantly differentially expressed between HFD and HFD-LFD. Pathway enrichment analysis revealed that also under basal conditions, the inflammatory response of PMs was affected by HFD (online supplementary figure S3D). Taken together, our data show that DIO and T2D restrict LPS-induced immune activation of PMs. Interestingly, this effect is already observed prior to LPS stimulation, so it seems that a HFD primes PMs towards a deactivated phenotype which is translated to an impaired LPS response.

To confirm this deactivated immune response, proinflammatory surface markers and cytokine secretion were measured of LPS-stimulated PMs. We found a significantly lower surface expression of costimulatory molecule CD86 and Fc receptor CD64 on HFD compared with LFD and HFD-LFD PMs (figure 3A), also after 10 weeks of HFD (online supplementary figure S4A). Moreover, CD64 expression was also significantly lower without LPS stimulation. HFD PMs secreted less TNF, IFN $\gamma$, and $\mathrm{NO}$, which was reversed after weight loss (figure 3B,C). Besides, NO secretion was also significantly decreased after 10 weeks of HFD (online supplementary figure S4B). Simultaneously, we found a significantly increased expression of the anti-inflammatory surface receptor transferrin (CD71) on HFD PMs after IL-4 stimulation (figure 3D). These results confirm that HFD primes PMs towards a less proinflammatory state and that this state is reversible by a diet shift.

\section{Weight loss reverses the obesity and T2D affected metabolism of PMs}

To gain more knowledge about the cause and mechanism of the deactivated PM phenotype, we applied computational strategies. First, upstream regulator prediction analysis was performed on the significantly differentially regulated genes of HFD versus LFD and HFD versus HFD-LFD PMs with and without LPS. Activation by antiinflammatory upstream regulator IL10R $\alpha$ and suppression of proinflammatory upstream regulators IFN $\alpha / \beta$ and CD38 were predicted to play a significant role in the anti-inflammatory phenotype of the cells (figure 4A). ${ }^{22}$

Next, we constructed a coexpression network with the transcriptional data of the three different groups of unstimulated PMs. WGCNA identified 18 modules of similar expression obtained by hierarchical clustering with adjacency-based dissimilarity (online supplementary figure S5A). The brown module appeared to have the strongest positive correlation with the incorporated experimental traits diet and bodyweight whereas the green and red modules showed the strongest negative correlation (figure 4B). Genes present in those three modules appeared to have the highest mean GS (figure 4C). Pathway enrichment analysis indicated that genes present in the brown module play a role in the immune system (figure 4D). Remarkably, genes of the green module showed enrichment for metabolic processes like lipid metabolism which was not demonstrated with pathway enrichment analysis on all significantly differentially regulated genes (figure $2 \mathrm{~F}$ ). Transcripts in the red module were, however, less significantly, also assigned to metabolic pathways. A heatmap of the significantly differentially regulated genes of these two metabolic pathways confirmed the HFD-induced metabolic changes (online supplementary figure S5B). Next, we found the GS for bodyweight in the green module to be highly correlated $(\mathrm{R}=0.83$ ) with the module membership (similarity of a gene to the module eigengene; Figure 4E). This demonstrates that genes significantly associating with bodyweight are also the most prominent transcripts of the green module. We found that protein-coding transcripts 
A

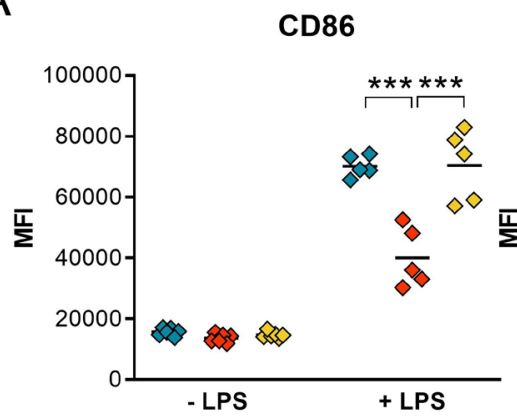

C

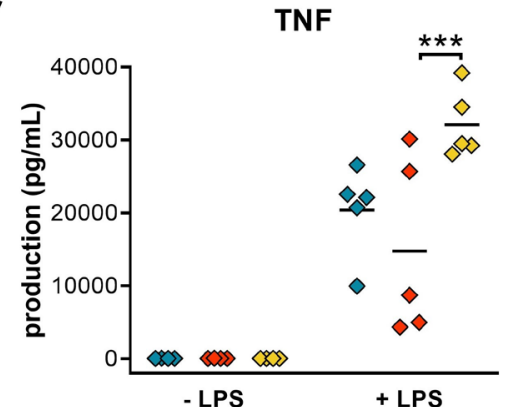

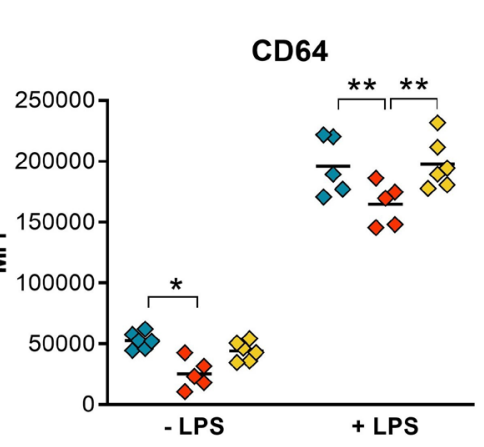

B

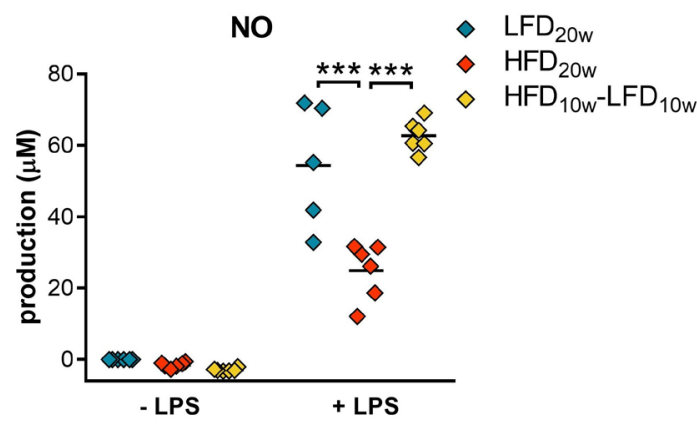

D CD71

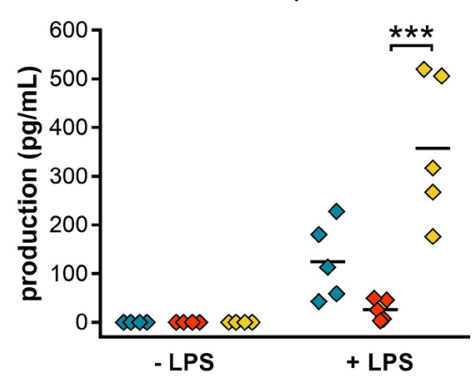

with a high GS and module membership of the green module form a significant protein-interaction network (figure 4F). This network encloses several V-type ATPases (ATP6V) and other proteins with metabolic, lysosomal and immune-related functions. Taken together, HFD affects PM metabolism which may play a role in the deactivated phenotype of PMs in obesity. We also found that this metabolic interruption directly correlates with bodyweight and disappears after a shift to LFD.

Spann et al described that a deactivated macrophage phenotype could be attributed to deregulated cholesterol synthesis pathways. ${ }^{23}$ Therefore, we next focused our analysis on these processes. Indeed, we found a significant downregulation of enzymes of the cholesterol biosynthesis, such as 3-hydroxy-3-methylglutaryl-CoA synthase 1 (Hmgcs1), lanosterol synthase (Lss), and 24-dehydrocholesterol reductase (Dhcr24) expression (online supplementary figure S5C). Dhcr24 catalyzes the reduction from desmosterol to cholesterol and its downregulation has been shown to lead to accumulation of desmosterol consequently activating liver $\mathrm{X}$ receptors (LXR) and sterol regulatory element-binding proteins (SREBP) target genes and suppressing inflammatory responses. In agreement, we found an upregulation of numerous LXR and SREBP targets in the HFD PMs, such as Idol, Srebf1, Srebf2, Scd1, and Abca1 (online supplementary figure $\mathrm{S} 5 \mathrm{C}$ ), as well as a downregulation of numerous proinflammatory genes (figure 2C). Furthermore, we found no clear activation or suppression of glycolysis and the pentose phosphate pathway (PPP).

\section{DISCUSSION}

Obesity and T2D affect the immune system and thereby play an important role in the pathophysiology of obesityrelated comorbidities like infection susceptibility. In this study, we focused on the effects of obesity and T2D on PMs since macrophages play a key role in defense against invading pathogens. ${ }^{24}$ For example, it has been shown that infection susceptibility to Mycobacterium tuberculosis and Streptococci spp correlates with a decreased NO and TNF production by macrophages. ${ }^{25} 26$ We also determined whether the HFD-induced effects were reversible after weight loss because it is not clear which obesityrelated complications will resolve after weight loss and which need additional treatment. Contrary to the overbalance of proinflammatory macrophages in adipose tissue in obesity and T2D, we demonstrate a deactivated state of macrophages outside the adipose tissue. Furthermore, we showed that this abnormally deactivated state of macrophages is lost after weight loss. Whether these deactivated macrophages are also present in patients with obesity and T2D and significantly contribute to their increased infection susceptibility remains to be determined. Our data suggest that weight loss in patients with obesity and T2D will restore the normal proinflammatory 
A
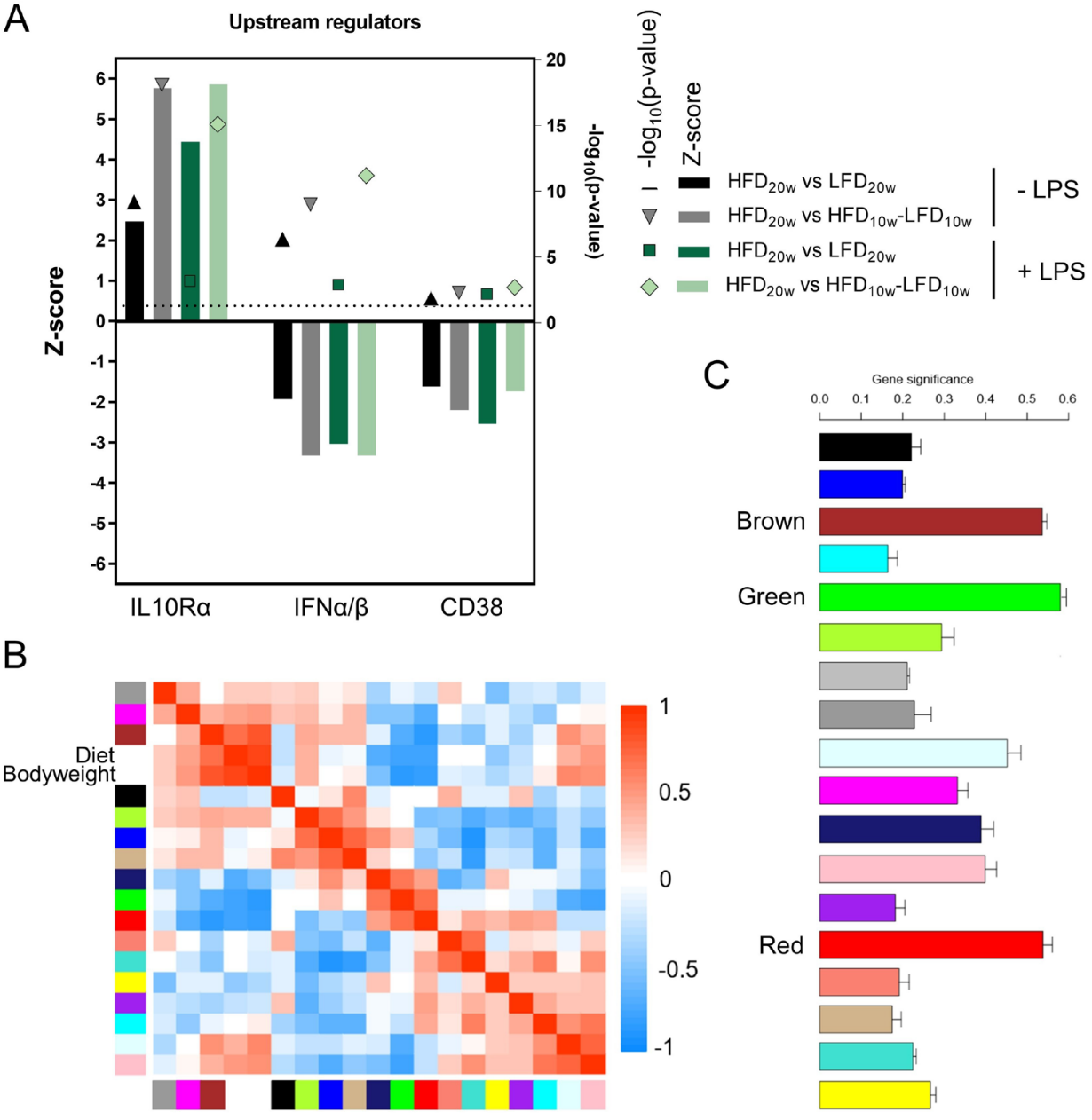

C
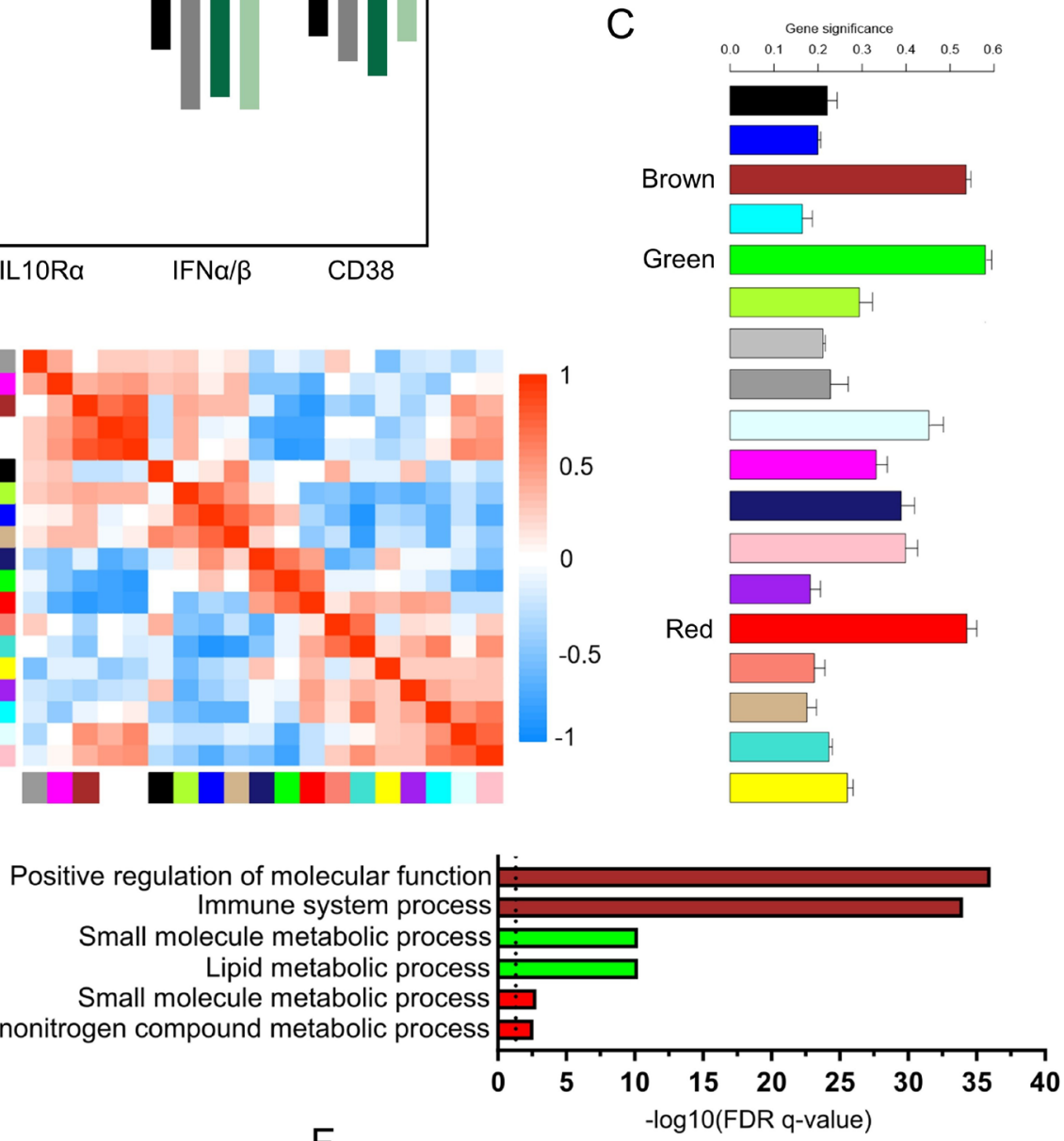

E

F
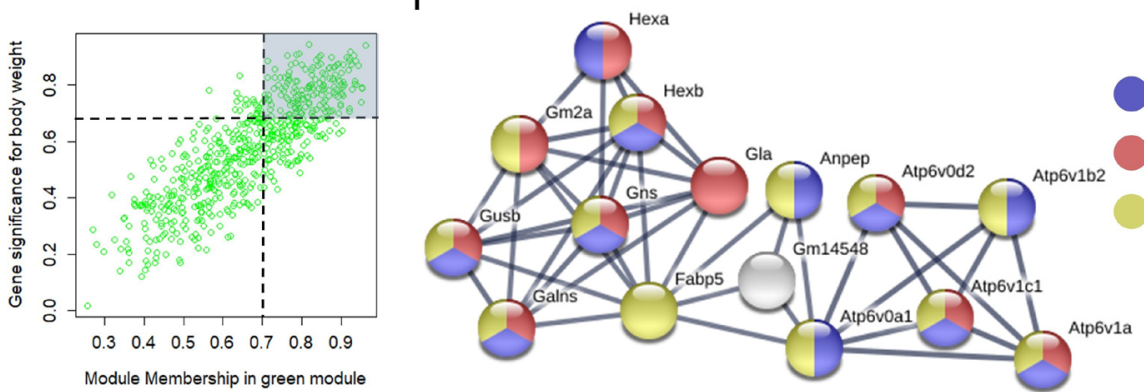

Metabolic pathways

Lysosome

Immune system

Figure 4 Peritoneal macrophage (PM) metabolism is affected by diet-induced obesity (DIO) and diabetes and is reversible by a shift to LFD. (A) Upstream regulator analysis of Ingenuity Pathway Analysis (IPA) on differentially regulated genes $(F D R<0.1)$. (B) Module eigengene adjacency heatmap. Module eigengenes were calculated by summarizing the first principal component of the modules. (C) Mean gene significance (GS) across all genes in a module. A large absolute value of the gene significance measure corresponds to a small two-sided P value. (D) Top five pathways of Gene Ontology pathway enrichment analysis of the brown, green, and red modules. (E) Scatter plot of the module membership in the green module against the GS for bodyweight. (F) Largest functional protein association network of the green module with high confidence (0.7). Hub genes with a module membership in the green module and an absolute GS for bodyweight $>0.7$ were selected for this network (162 genes). FDR, false discovery rate; HFD, high-fat diet; IFN, interferon; IL10, interleukin-10; LFD, low-fat diet; LPS, lipopolysaccharide. 
state of macrophages and thereby reduce immunerelated comorbidities of those patients.

First, the presence and reversibility of obesity and T2D were confirmed in the different groups. As expected, mice showed obesity and T2D characteristics (eg, weight gain, insulin resistance and high levels of plasma cholesterol and triglycerides) after 10 and 20 weeks of HFD. Besides, systemic low-grade inflammation, a classical hallmark and confounding factor for comorbidities, was confirmed in HFD mice by detection of elevated plasma TNF, CXCL1 and IL-10 levels, more infiltrating immune cells together with hepatic lipid accumulation, and enlarged adipocytes. van der Heijden et al also found significantly higher plasma levels of TNF, CXCL1, and IL-10 in obese mice. ${ }^{27}$ In our studies, all changes disappeared after weight loss and our results support that the HFD induces a chronic inflammatory state which is reversible by dietary changes.

Transcriptional profiling indicated that DIO and T2D affected PM activation. We found a downregulation of proinflammatory genes and upregulation of antiinflammatory genes in HFD PMs. Significantly lower surface expression levels of CD86 and CD64 on HFD PMs and less secretion of TNF, IFN $\gamma$ and NO confirmed the deactivated PM state. Simultaneously, HFD PMs expressed significantly increased levels of CD71 after IL-4 incubation. All these differences disappeared after the diet shift. Thus, our data clearly show that a HFD dampens the LPS response of PMs and this effect disappears after weight loss. Interestingly, these changes are also observed before LPS stimulation, so it seems that PMs are tolerized by the HFD. In line with our findings, Amar et al showed that Porphyromonas gingivalis exposed PMs from mice with DIO produced less TNF, IL-1 $\beta$, and IL-6. ${ }^{28}$ Similarly, Ieronymaki et al demonstrated that alveolar macrophages from insulin-resistant mice produced significantly less TNF after LPS exposure and expressed more arginase $1 .{ }^{29}$ They also showed a reduced inflammatory response after a cecal ligation and puncture (polymicrobial sepsis model) in diabetic mice.

Several computational strategies were applied to unravel the cause of the deactivated PM phenotype. Upstream regulator prediction analysis predicted an upregulation of upstream regulator IL10R $\alpha$ and a downregulation of IFN $\alpha / \beta$ and CD38 coinciding with the less inflammatory phenotype of HFD PMs. Indeed, we and others found higher plasma IL-10 levels in obese mice, which can trigger the IL-10 receptor and induce a deactivated macrophage phenotype. ${ }^{30}$ Second, type 1 IFNs protect the host from viral infections and induce myeloid-mediated bacterial clearance. $^{31}{ }^{32}$ Furthermore, Matalonga et al found that the number of infected macrophages and intracellular bacteria was increased in CD38-deficient macrophages, which coincides with the predicted downregulation of CD38 and a flawed LPS response in HFD PMs. ${ }^{33}$ Notwithstanding, other factors, or a combination of factors, like the richly present unsaturated fatty acid oleic acid of the HFD may also contribute to the deactivated macrophage phenotype. $^{3435}$
WGCNA identified HFD-induced metabolic alterations to correlate with bodyweight. These metabolic changes may play a role in the mechanism of the deactivated phenotype since metabolism and macrophage function are highly dependent. ${ }^{36}$ For instance, it has been shown that T2D promotes an impaired control of $M$. tuberculosis in human foamy macrophages by inducing lysosomal dysfunction. ${ }^{37}$ Additionally, previous work has shown that PMs from $\mathrm{Ldll}^{-/-}$mice fed a Western diet show a deactivated phenotype. ${ }^{23} 38$ These macrophages differentiate in a cholesterol-rich and atherosclerotic environment and will become lipid-laden foam cells. Their deactivated phenotype is explained by the accumulation of desmosterol $^{23}$ and a disrupted PPP. ${ }^{38}$ In our current studies, we have focused on a DIO model with insulin resistance and in this model, it has been shown that PMs do not acquire foam cell characteristics. ${ }^{39}$ Similarly, as we demonstrated in our mice, patients with obesity and T2D may also encounter immune-related complications in the absence of atherosclerosis and hypercholesterolemia. Despite the absence of foam cells, we hypothesize that LXRdependent and SREBP-dependent mechanisms might explain our deactivated PM phenotype. In accordance with Spann $e t$ al, we found a significant downregulation of several enzymes of the cholesterol biosynthesis pathway, including Dhcr $24 .^{23}$ Downregulation of Dhcr24 has been shown to result in accumulation of desmosterol which can activate LXR and SREBP target genes. Furthermore, it has been shown that LXR activation inhibits inflammatory responses. In agreement with Spann $e t$ al, we found an upregulation of numerous LXR and SREBP targets in the HFD PMs, as a downregulation in numerous proinflammatory genes. Thus, LXR-dependent and SREBPdependent mechanisms may explain our deactivated PM phenotype.

Inflammation plays a role in host defense against pathogens and impacts the development and progression of cancer. Michelet et al recently found that obesity also limits the antitumor response of natural killer cells in mouse and human. ${ }^{40}$ Furthermore, tumor-associated macrophages (TAM) have anti-inflammatory characteristics that promote tumor immune evasion and maintain tumors by specific cytokine secretion that increases tumor cell viability and facilitates metastasis. ${ }^{41}$ On the other hand, proinflammatory macrophages have been shown to inhibit tumor growth and induce cell death. For instance, the CD86-CD28 interaction has been shown to play an important role in the induction of T-cell-mediated tumor killing. Moreover, low numbers of $\mathrm{CD} 86^{+}$TAMs have been correlated with an aggressive tumor phenotype in patients. ${ }^{42}$ Since we identified antiinflammatory macrophages in obesity, with significantly lower CD86 expression, and anti-inflammatory macrophages are known to significantly contribute to cancer progression, we speculate that these macrophages may affect cancer progression in patients and that weight loss will be beneficial in this respect as well. 
Hyperglycemic memory is a term that has been used to describe the occurrence that despite maintaining normal blood glucose levels after a period of hyperglycemia, a subset of the patients with diabetes develops comorbidities nonetheless. ${ }^{43-45}$ This hyperglycemic memory phenomenon can be a result of epigenetic changes caused by hyperglycemia which persist after normoglycemia. ${ }^{46}$ For example, Rodrigues et al showed with $\mathrm{db} / \mathrm{db}$ mice that T2D can result in stem cell aberrations which directly affect tissue function and persist after return to normoglycemia. ${ }^{9}{ }^{47}$ Still, Siersbæk et al, who used a model more similar to ours, found that the HFD-induced changes of mouse hepatic transcription and enhancer activity were reversible after weight loss, which is more consistent with our findings. ${ }^{48}$ Future research should focus on hyperglycemic memory and determine when this phenomenon is existing and when not. This will be very useful for the treatment of obese individuals with T2D and follow-up care after the maintenance of normal blood glucose levels.

In summary, contrary to the proinflammatory phenotype of macrophages in adipose tissue in obesity and T2D, we demonstrate a deactivated state of macrophages outside the adipose tissue. Suppressed macrophage activity may contribute to the impaired immune response against pathogens and increased prevalence of infections that are seen in obese and T2D individuals. Finally, we show beneficial effects of weight loss in these settings by reversal of the deactivated macrophage phenotype.

\section{Author affiliations \\ ${ }^{1}$ Experimental Vascular Biology, Department of Medical Biochemistry, Amsterdam Cardiovascular Sciences (ACS), Amsterdam UMC-Location AMC, University of Amsterdam, Amsterdam, Netherlands \\ ${ }^{2}$ Departments of Pathology and Molecular Genetics, CARIM School for Cardiovascular Diseases and GROW School for Oncology and Developmental Biology, Maastricht University, Maastricht, Netherlands \\ ${ }^{3}$ Institute for Cardiovascular Prevention (IPEK), Ludwig Maximilians University Munich, Munich, Germany}

Contributors LW, AEN, EL, and MPJW designed the research. LW performed the majority of experiments with contributions from AEN (flow cytometry), SV, MT, CPAAR, and MER (glucose and insulin tolerance tests), SV (intraperitoneal injections, blood processing and flushing peritoneum) and MJJG (organ isolation). LW and MJJG scored tissue sections. LW and GRG performed RNA-seq library preparation. LW analyzed the data. KHMP helped with the bioinformatics. LW wrote the manuscript and MPJW, SV, AEN, MJJG, MER, EL, and KHMP gave feedback. MPJW supervised the study. MPJW is the guarantor of this work and, as such, had full access to all the data of the study and takes responsibility for all the integrity of the data and accuracy of the data analysis.

Funding This work was supported by The Netherlands Heart Foundation (CVON 2011/B019, CVON 2017-20); Spark-Holding (2015B002); the European Union (ITN grant EPIMAC); Leducq Foundation (Transatlantic Network Grant); REPROGRAM (EU Horizon 2020); and AMC Fellowship.

Competing interests None declared.

Patient consent for publication Not required.

Ethics approval Mice were housed at the Animal Research Institute Amsterdam UMC (ARIA). All animal procedures were approved by the ethical committee for animal welfare of the Amsterdam UMC.

Provenance and peer review Not commissioned; externally peer reviewed.

Data availability statement Data are available upon reasonable request.
Open access This is an open access article distributed in accordance with the Creative Commons Attribution 4.0 Unported (CC BY 4.0) license, which permits others to copy, redistribute, remix, transform and build upon this work for any purpose, provided the original work is properly cited, a link to the licence is given, and indication of whether changes were made. See: https://creativecommons.org/ licenses/by/4.0/.

ORCID IDs

Lisa Willemsen http://orcid.org/0000-0002-9676-9249

Myrthe E Reiche http://orcid.org/0000-0002-9295-6418

Guillermo R Griffith http://orcid.org/0000-0002-3983-1499

Menno P J de Winther http://orcid.org/0000-0002-4038-6636

\section{REFERENCES}

1 Haslam DW, James WPT. Obesity. The Lancet 2005;366:1197-209.

2 Hegde V, Dhurandhar NV. Microbes and obesity--interrelationship between infection, adipose tissue and the immune system. Clin Microbiol Infect 2013;19:314-20.

3 Falagas ME, Kompoti M. Obesity and infection. Lancet Infect Dis 2006;6:438-46.

4 Zulkipli MS, Dahlui M, Jamil N, et al. The association between obesity and dengue severity among pediatric patients: a systematic review and meta-analysis. PLoS Negl Trop Dis 2018;12:1-22.

5 Wyss K, Wångdahl A, Vesterlund M, et al. Obesity and diabetes as risk factors for severe Plasmodium falciparum malaria: results from a Swedish nationwide study. Clin Infect Dis 2017;65:949-58.

6 Maier HE, Lopez R, Sanchez N, et al. Obesity increases the duration of influenza A virus shedding in adults. $J$ Infect $D$ is 2018;218:1378-82.

7 Wing RR, Lang W, Wadden TA, et al. Benefits of modest weight loss in improving cardiovascular risk factors in overweight and obese individuals with type 2 diabetes. Diabetes Care 2011;34:1481-6.

8 Hernandez-Carretero A, Weber N, La Frano MR, et al. ObesityInduced changes in lipid mediators persist after weight loss. Int $J$ Obes 2018;42:728-36.

9 Januszyk M, Sorkin M, Glotzbach JP, et al. Diabetes irreversibly depletes bone marrow-derived mesenchymal progenitor cell subpopulations. Diabetes 2014;63:3047-56.

10 Shoelson SE, Herrero L, Naaz A. Obesity, inflammation, and insulin resistance. Gastroenterology 2007;132:2169-80.

11 Daryabor G, Kabelitz D, Kalantar K. An update on immune dysregulation in obesity-related insulin resistance. Scand J Immunol 2019;89:e12747.

12 Boutens L, Stienstra R. Adipose tissue macrophages: going off track during obesity. Diabetologia 2016;59:879-94.

13 Lee YS, Li P, Huh JY, et al. Inflammation is necessary for long-term but not short-term high-fat diet-induced insulin resistance. Diabetes 2011;60:2474-83.

14 Dobin A, Davis CA, Schlesinger F, et al. Star: ultrafast universal RNAseq aligner. Bioinformatics 2013;29:15-21.

$15 \mathrm{Li} \mathrm{H}$, Handsaker B, Wysoker A, et al. The sequence Alignment/Map format and SAMtools. Bioinformatics 2009;25:2078-9.

16 Heinz S, Benner C, Spann N, et al. Simple combinations of lineage-determining transcription factors prime cis-regulatory elements required for macrophage and $\mathrm{B}$ cell identities. Mol Cell 2010;38:576-89.

17 Love MI, Huber W, Anders S. Moderated estimation of fold change and dispersion for RNA-Seq data with DESeq2. Genome Biol 2014;15:1-21.

18 Zhou Y, Zhou B, Pache L, et al. Metascape provides a biologistoriented resource for the analysis of systems-level datasets. Nat Commun 2019;10.

19 Langfelder P, Horvath S. WGCNA: an R package for weighted correlation network analysis. BMC Bioinformatics 2008;9:559.

20 Zhang B, Horvath S. A general framework for weighted gene co-expression network analysis. Stat Appl Genet Mol Biol 2005;4:Article17.

21 Szklarczyk D, Franceschini A, Wyder S, et al. String v10: proteinprotein interaction networks, integrated over the tree of life. Nucleic Acids Res 2015;43:D447-52.

22 Amici SA, Young NA, Narvaez-Miranda J, et al. Cd38 is robustly induced in human macrophages and monocytes in inflammatory conditions. Front Immunol 2018;9:1593.

23 Spann NJ, Garmire LX, McDonald JG, et al. Regulated accumulation of desmosterol integrates macrophage lipid metabolism and inflammatory responses. Cell 2012;151:138-52.

24 Zhang L, Wang C-C. Inflammatory response of macrophages in infection. Hepatobiliary Pancreat Dis Int 2014;13:138-52. 
25 Braverman J, Sogi KM, Benjamin D, et al. HIF-1 $\alpha$ Is an Essential Mediator of IFN- $\gamma$-Dependent Immunity to Mycobacterium tuberculosis. J Immunol 2016;197:1287-97.

26 Knight M, Stanley S. HIF- $1 \alpha$ as a central mediator of cellular resistance to intracellular pathogens. Curr Opin Immunol 2019;60:111-6.

27 van der Heijden RA, Sheedfar F, Morrison MC, et al. High-Fat diet induced obesity primes inflammation in adipose tissue prior to liver in C57BL/6J mice. Aging 2015;7:256-68.

28 Amar S, Zhou Q, Shaik-Dasthagirisaheb Y, et al. Diet-Induced obesity in mice causes changes in immune responses and bone loss manifested by bacterial challenge. Proc Natl Acad Sci U S A 2007;104:20466-71.

29 leronymaki E, Theodorakis EM, Lyroni K, et al. Insulin resistance in macrophages alters their metabolism and promotes an M2-like phenotype. J Immunol 2019;202:1786-97.

30 Mantovani A, Sica A, Sozzani S, et al. The chemokine system in diverse forms of macrophage activation and polarization. Trends Immunol 2004;25:677-86.

31 Thomas KE, Galligan CL, Newman RD, et al. Contribution of interferon-beta to the murine macrophage response to the Toll-like receptor 4 agonist, lipopolysaccharide. J Biol Chem 2006;281:31119-30.

32 Eshleman EM, Lenz LL. Type I interferons in bacterial infections: taming of myeloid cells and possible implications for autoimmunity. Front Immunol 2014;5:1-11.

33 Matalonga J, Glaria E, Bresque M, et al. The nuclear receptor LXR limits bacterial infection of host macrophages through a mechanism that impacts cellular NAD metabolism. Cell Rep 2017;18:1241-55.

34 Camell C, Smith CW. Dietary oleic acid increases M2 macrophages in the mesenteric adipose tissue. PLoS One 2013;8:1-10.

35 Palomer X, Pizarro-Delgado J, Barroso E, et al. Palmitic and oleic acid: the yin and yang of fatty acids in type 2 diabetes mellitus. Trends Endocrinol Metab 2018;29:178-90.

36 Wang S, Liu R, Yu Q, et al. Metabolic reprogramming of macrophages during infections and cancer. Cancer Lett 2019;452:14-22.
37 Vrieling F, Wilson L, Rensen PCN, et al. Oxidized low-density lipoprotein (oxLDL) supports Mycobacterium tuberculosis survival in macrophages by inducing lysosomal dysfunction. PLoS Pathog 2019;15:e1007724

38 Baardman J, Verberk SGS, Prange KHM, et al. A defective pentose phosphate pathway reduces inflammatory macrophage responses during hypercholesterolemia. Cell Rep 2018;25:2044-52.

39 Reardon CA, Lingaraju A, Schoenfelt KQ, et al. Obesity and insulin resistance promote atherosclerosis through an IFN $\gamma$-Regulated macrophage protein network. Cell Rep 2018;23:3021-30.

40 Michelet X, Dyck L, Hogan A, et al. Metabolic reprogramming of natural killer cells in obesity limits antitumor responses. Nat Immunol 2018;19:1330-40.

41 Corrêa LH, Corrêa R, Farinasso CM, et al. Adipocytes and macrophages interplay in the orchestration of tumor microenvironment: new implications in cancer progression. Front Immunol 2017;8:1-12.

42 Dong P, Ma L, Liu L, et al. CD86+/CD206+, diametrically polarized tumor-associated macrophages, predict hepatocellular carcinoma patient prognosis. Int J Mol Sci 2016;17:320-12.

43 Tonna S, El-Osta A, Cooper ME, et al. Metabolic memory and diabetic nephropathy: potential role for epigenetic mechanisms. Nat Rev Nephrol 2010;6:332-41.

44 Engerman RL, Kern TS. Progression of incipient diabetic retinopathy during good glycemic control. Diabetes 1987;36:808-12.

45 Roy S, Sala R, Cagliero E, et al. Overexpression of fibronectin induced by diabetes or high glucose: phenomenon with a memory. Proc Natl Acad Sci U S A 1990;87:404-8.

46 Cencioni C, Spallotta F, Greco S, et al. Epigenetic mechanisms of hyperglycemic memory. Int J Biochem Cell Biol 2014;51:155-8.

47 Rodrigues M, Wong VW, Rennert RC, et al. Progenitor cell dysfunctions underlie some diabetic complications. Am J Pathol 2015;185:2607-18

48 Siersbæk M, Varticovski L, Yang S, et al. High fat diet-induced changes of mouse hepatic transcription and enhancer activity can be reversed by subsequent weight loss. Sci Rep 2017;7:40220. 